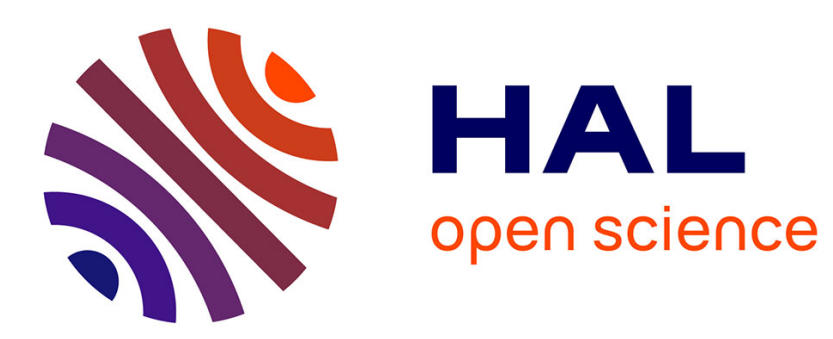

\title{
Limb positioning and shear flows in tetrapods
}

O.P. Boryskina, A. Al-Kilani, V. Fleury

\section{- To cite this version:}

O.P. Boryskina, A. Al-Kilani, V. Fleury. Limb positioning and shear flows in tetrapods. European Physical Journal: Applied Physics, 2011, 55 (2), 10.1051/epjap/2011100468 . hal-00723604

\section{HAL Id: hal-00723604 \\ https://hal.science/hal-00723604}

Submitted on 11 Aug 2012

HAL is a multi-disciplinary open access archive for the deposit and dissemination of scientific research documents, whether they are published or not. The documents may come from teaching and research institutions in France or abroad, or from public or private research centers.
L'archive ouverte pluridisciplinaire HAL, est destinée au dépôt et à la diffusion de documents scientifiques de niveau recherche, publiés ou non, émanant des établissements d'enseignement et de recherche français ou étrangers, des laboratoires publics ou privés. 


\title{
Limb positioning and shear flows in tetrapods.
}

\author{
Olena P. Boryskina+, Alia Al-Kilani* and Vincent Fleury*\$ \\ +Institute of Radiophysics and Electronics NAS of Ukraine, Acad. Proskura str. 12, Kharkov \\ 61085, Ukraine \\ *Laboratoire Matière Systèmes Complexes \\ 10 rue Alice Domon et Léonie Duquet \\ CNRS/Université Paris-Diderot \\ Paris 75013 France
}

\begin{abstract}
.
There is increasing evidence that animal morphogenesis consists of a large scale tissue flow, which defines the gross characteristics of the animal body at a very early developmental stage. We have studied the vertebrate embryo cell trajectories between a moment when it is flat and formless, to a moment when the body plan is recognizable (chicken embryo days 2-3 of development). We find that a large vortex flow patterns the vertebrate bauplan, and especially the limb territories, both hindlimbs and forelimbs. In vivo velocity measurements show that the vortices are dragged by a localized shear oriented along the median axis. A simple hydrodynamic model accounts for the lenticular shape of the limb plates. On the hindlimb plate, the flow propagates in the form of a solidbody vortex on the limb plate, dragged by a Poiseuille flow along the backbone. In vivo tonometry measurement shows that there exist stress gradients in the embryonic tissue, and that the flow pattern is congruent with the direction of decrease of stress magnitude.
\end{abstract}

PACS Numbers : 87.00.00, 87.19.1x, 87.18.Hf, 87.19.1p

$\S$ Author for correspondence 


\section{I.Introduction.}

It has been suggested that, at very early stages, embryonic material is viscous and that hydrodynamic laws of viscous flows may apply to the formation of the pattern of embryos [14]. These hydrodynamic laws should help to understand the vortices which are observed in vertebrate development [5,6]. Especially, in 2D tissue sheets, localized oriented flows of cells induce naturally large scale vortices, by mass conservation of the fluid [1]. While spatially extended genetic feedbacks are always possible, considering the complexity of signalling pathways, the laws of physics make it unnecessary that such genetic feedback loops pattern explicitely the fluid speed point by point at all points. By physical laws, a localized pull in a continuous medium suffices to induce long range effects, such as large scale vortices, thereby making morphogenesis easier to control globally by genetic expressions. Therefore, it is possible that different animal bauplans (viewed as the global mechanical attractor) are determined by the topology of localized early (initial conditions) patterns of expressions, which are upscaled by the physics of continuum materials.

In this respect, several articles have confirmed recently that there is very little chemotaxis during embryo development, and that vertebrate embryo development is a mass flow $[7,8]$. This confims classical work about the fluid character of biological tissue sheets $[9,10]$. In the continuum material limit, and in such a slow dynamics, thin film elasto-plastic flow can be considered as a creeping viscous flow of tissue [1]. The topology of the flow is dictated by the initial symmetry breaking in the starting condition. The early structure in the embryo blastula suggests that the initial flow forms a hyperbolic flow of cells, composed of large vortices revolving around a hyperbolic point (stagnation point).

The initial vortices at the blastula stage, the initial hyperbolic flow and the stagnation point have been observed for long at a qualitative level in the chicken [5]. We address here a later stage, as the body forms. We show that such vortex flows (i.e., streamlines exhibiting closed loops) continue during all stages of embryo morphogenesis, until a vertebrate bauplan starts to be recognizable. In this perspective, we confirm that the limb areas actually form dynamically as a consequence of the vortex flows [2-4], because of the strong winding of the tissue sheared along the median axis. The latter behaves as a central elongated element which 
extends and drags the lateral tissue. In this article we show experimentally that there is indeed a correlation between the rotation of the flow and the positioning of the limb area, associated with stress gradients.

This article is divided into parts as follows. In part II, we show the evidence of vortex-type flows, with a singular point at the apex of the primitive streak during tetrapod morphogenesis[11]. In part III we explain why such a hyperbolic flow appears at the apex of the furrow. Then (part IV) we propose a simple hydrodynamic modelling of this flow. This modelling predicts the existence of an oval rotating core, located along the line of pull, as observed. This core experiences a high shear. We show that the physical boundary of the hindlimb field appears exactly in the core of the vortex. Next, we show that, after the physical boundary has formed, the median axis takes the shape of a central elongated element which stirs the hindlimb area, with a very simple dynamic pattern: the median axis extends as a Poiseuille flow, and the lateral plates revolve as a solid-body vortex. Between the two geometrical domains there is a physical discontinuity.

Finally, in part $\mathbf{V}$, We present in vivo tonometry measurements showing that there is a pressure gradient oriented as predicted from the flow pattern. The materials and methods are presented in Appendix.

\section{Direct experimental observations of vortices during vertebrate embryo morphogenesis.}

At the end of the blastula stage, the embryo is composed of a flat tissue, with a furrow in the middle (Figure 1). The embryo body is not formed yet. The apex of the furrow (called Hensen's node) starts to extend "downwards" (i.e. caudally) along the Antero-Posterior axis, and to "induce" the body parts. We have studied the embryo cell trajectories at this stage. Actually, the process of embryo formation, as the time-lapse movies show, is a physical vortex movement of the entire tissue (Figures 2-7) with a long range coherent organization. PIV tracking of the tissue movement at the stage of Fig. 1, and over the next hours, shows that the movement of the furrow shears the lateral tissue, and generates a pair of large vortices, that descends caudally together with the recession of the apex (Figs. 2,3). The vortices are oval and stretch along the lateral sides of the median axis, as the median axis extends. (It is also observed that there is a small delay, ca 30 minutes, between the right and left half, which may possibly be ascribed to molecular chirality of epithelial cillia [12] which bias cellular traffic). Also, smaller vortices, or maybe only fraction of vortices, are generated by the extension, in the 
presumptive shoulders area, which move in the opposite direction (these are visible in the top of Figs. 2,3). Actually, the anterior vortices are perturbed by the forward movement of the head which passes (again by folding) over the blastula plane, and then overhangs. It is known that at previous stages, when the blastula starts to revolve, there are large vortices rotating in the anterior direction, we therefore conclude that the superimposition of a new large caudal shear, over an already existing large cranial rotation, forms a hyperbolic pattern of rotation as we observe, with flows oriented in both directions.

The sequence of plates (Figs. 2-7) shows the lower half of the body as it extends caudally. It shows very clearly that the formation of the lower half of the body is driven by the median axis shear, which stirs a large vortex flow sideways (two vortices forming a vortex dipole). The cores of the two large vortices located in the posterior area migrate caudally (Movies 1-5 [11]) at a speed of the order of 1 microns /min. The map of $\operatorname{div}(\mathbf{V})$ at an early stage as the one in Fig. 1 shows an almost flat landscape, with nevertheless a small departure from flatness in the region of the apex of the furrow, where a pull is exerted upon the median axis, just ahead of the apex of the primitive streak. The chord formation along the median axis is associated to this pull (see esp. Movie 2).

While the tissue in the area of the presumptive hindlimbs rotates, a lenticular bulging forms, which is progressively separated from the median axis by a very clear line (Fig. 6). This singularity is associated to the future hindlimb area and is found exactly in the area of highest shear of the revolving vortex core (Figs. 9, 10). The line follows the edge of the flank (the line is especially conspicuous in Figs. 3 and 6). When the line of singularity is inspected very carefully, one observes that it forms by propagating a fold across the vortex core, from the posterior end of the vortex core (apex of the singularity) to the anterior end of the vortex core (presumptive navel area), this movement accompanies the collision of the lateral flow of the vortex against the unidirectional movement along the median axis.

As the presumptive pelvic area becomes more recognizable, the large circular flow in this area is permanently evidenced (Figs. 5-7). It moves caudally, and the winding of the tissue forms the precursor of the pelvic iliac bone, as well known in anatomy (please refer to an atlas of vertebrate anatomy for an anatomical description of the pelvis). At the distal (most caudal) end of the pelvis, in the pubic area, the movement is away from the median axis, and it therefore tends to open asunder the left and right part of the pubic area, as observed also at an anatomic level (the so-called falciform processes move asunder). 


\section{Explanation of the hyperbolic structure of the ectodermal flow.}

It is known that the blastula movement has two phases : during a first phase, it revolves in plane, towards a hyperbolic point located posteriorily[1]. During a second phase, the top layer invaginates, and it now revolves with a hyperbolic point also, but shifted at the apex of the hole through which invagination occurs. There is a straightforward physical explanation to the establishment of a second hyperbolic flow with a singularity located more anterior to the apex of the furrow. In the first phase, the ectoderm being attracted caudally generates a hyperbolic vector field singularity in the posterior area [1]. At this singular point, the tissue invaginates (sort of escape of the singularity). But this invagination causes a $\mathrm{U}$ turn in the vertical plane (Fig. 11). As a consequence, a situation of tank treading is created by which an additional traction force, due to the underneath layer, is added to the forces acting on the top layer. This strong pull, by the newly appeared underneath layer, is added at the apex of the furrow upon the top layer which was revolving anteriorily. Physically, this adds a dipole oriented posteriorily, and thus shifts the hyperbolic point to a position ahead of the apex of the furrow. This explains the global hyperbolic pattern of the ectoderm.. Why the initial flow has a hyperbolic character is still a matter of debate, but physical arguments explain clearly the shift in the position of the hyperbolic point.

To summarize the sections II-III, we have shown that the extension of the median axis shears the entire embryo, and generates hyperbolic streamlines along the sides of the dorsal axis which co-localize with the limb territories. Complete loops are evidenced in the hindlimb area at all stages up to at least 8 somites stage. In the forelimb area, it is known that loops are complete before the stages described here, while we find incomplete loops. This can be ascribed to the forward extension of the head over the blastula plane.

The shear along the median axis which generates large "vortex" flows induces a bulging in the area of the presumptive hindlimbs and pelvis, and in the area of the forelimbs.

During this bulging a line of tissue discontinuity (fold) appears, that segregates the axis of the body and the limbs territories. In the hindlimb zone, this line forms in about $30 \mathrm{~min}$. a physical boundary between the median axis (which shows a more unidirectional flow) and a large revolving area forming the presumptive pelvic and limb area (which shows circular streamlines). This hyperbolic flow situation implies a partial miror symmetry between the upper and lower part of the body, as described in detail in 1868 by Wyman [13]. This paleontologist reviewed all the accumulated data of his time, to show that animals were not 
built upon a plan organized in the head-to-tail direction, but more likely in a plan having mirror symmetry with respect to the center of the body. Also, he ascribed this organization to a mirror symmetry in the vector fields of development, which he described as analogous to dipolar magnetic fields. Such a broken symmetry may well suffice to explain the opposite chirality of upper and lower limbs. In this view, the mere advection of the entire embryo domain in a hyperbolic pattern would break the symmetry of all concentration fields, thereby generating organs with a partial top-bottom symmetry, in addition to the usual left-right symmetry. In this view, the formation of the animal body amounts qualitatively to an up-scaling of the initial hyperbolic flow pattern towards the final animal shape, which inherits and conserves at all stages the global pattern [4].

\section{Mathematical modelling}

It has been observed that at the stage shown in Fig. 1, all genetic markers are very localized along the Antero-Posterior axis, because of the previous embryo contraction ( Ref. 4). This is especially true of all known growth factors, which are concentrated along the median axis by the flow itself. Not only the force term is concentrated at the median axis, but the movement itself, but its hyperbolic character, tends to stretch the pattern along the median axis, and smoothen the gradients. As a consequence, we expect the volume force terms for the flow (produced by cell swelling and traction) to be concentrated along the Antero-Posterior axis. Typical in vivo measurements of the fluid speed show that the flow starts by a strong pull centred along the Antero-Posterior axis (Fig. 9Left), which diffuses laterally to make the vortex revolve (Fig. 9Right). Therefore, we may expect that the pattern of movement is the one generated by a pulling rectangle, centered along the median axis, and shearing the lateral tissue. We can calculate the pattern of flow in the following way. The stream function generated in a viscous plane by a moving dipole located at position $(\alpha, \beta)$ is [1]:

$$
\left.\Psi_{0}(\mathrm{x}, \mathrm{y}, \alpha, \beta)=(\mathrm{x}-\alpha) /\left[(\mathrm{x}-\alpha)^{2}+(\mathrm{y}-\beta)^{2}\right)\right] \quad \text { Equ. } 1
$$

which corresponds to an in-plane vector field :

$$
\mathbf{V}(\mathrm{x}, \mathrm{y})=\mathrm{u}_{0} \operatorname{curl}\left(0,0 \Psi_{0}(\mathrm{x}, \mathrm{y}, \alpha, \beta)\right)=\left(\partial_{\mathrm{y}} \Psi_{0}(\mathrm{x}, \mathrm{y}, \alpha, \beta),-\partial_{\mathrm{x}} \Psi_{0}(\mathrm{x}, \mathrm{y}, \alpha, \beta), 0\right) \quad \text { Equ. } 2
$$

where $\left(0,0, \Psi_{0}(x, y, \alpha, \beta)\right)$ is the potential vector of the fluid, and $\mathrm{u}_{0}$ a dimensioning factor proportional to the magnitude of the pulling force of the dipoles. The movement generated by a rectangle of pulling cells bounded by two sides of length $2 \mathrm{~B}$ and two sides of length $2 \mathrm{~L}$ is 
found by integrating Equ. 1 between $-\mathrm{B}$ and $+\mathrm{B}$ over the variable $\beta$, and between $-\mathrm{L}$ and $\mathrm{L}$ over $\alpha$, wich gives the expression :

$\Psi_{\text {total }}(\mathrm{x}, \mathrm{y}, \mathrm{L}, \mathrm{B})=(\mathrm{y}-\mathrm{B}) \operatorname{Arg} \operatorname{cotan}[(\mathrm{y}-\mathrm{B}) /(\mathrm{x}-\mathrm{L})]+[(\mathrm{x}-\mathrm{L}) / 2] \ln \left[(\mathrm{x}-\mathrm{L})^{2}+(\mathrm{y}-\mathrm{B})^{2}\right]-(\mathrm{y}-$

$\mathrm{B}) \operatorname{Arg} \operatorname{cotan}[(\mathrm{y}-\mathrm{B}) /(\mathrm{x}+\mathrm{L})]+[(\mathrm{x}+\mathrm{L}) / 2] \ln \left[(\mathrm{x}+\mathrm{L})^{2}+(\mathrm{y}-\mathrm{B})^{2}\right] \quad-\{(\mathrm{y}+\mathrm{B}) \operatorname{Arg} \operatorname{cotan}[(\mathrm{y}+\mathrm{B}) /(\mathrm{x}-$

$\mathrm{L})]+[(\mathrm{x}-\mathrm{L}) / 2] \ln \left[(\mathrm{x}-\mathrm{L})^{2}+(\mathrm{y}+\mathrm{B})^{2}\right]-(\mathrm{y}+\mathrm{B}) \operatorname{Arg} \operatorname{cotan}[(\mathrm{y}+\mathrm{B}) /(\mathrm{x}+\mathrm{L})]+[(\mathrm{x}+\mathrm{L}) / 2] \ln \left[(\mathrm{x}+\mathrm{L})^{2}+(\mathrm{y}+\mathrm{B})\right.$

$\left.\left.{ }^{2}\right]\right\}$

Equ. 3

This gives the vortex shown in Fig. 12. We indeed find an elongated, oval, vortex dipole, with a point-like feature at the lateral extremities of the vortex. The actual aspect ratio of the oval is determined by the length of the pulling area. It makes sense that the oval stretches as more cells invaginate along the furrow. The purpose of this calculation is to show that it is not necessary to invoke complex genetic inductions, to find such an oval shape stretching along an elongated central shearing element; very simple hydrodynamic physics is able to form long-ranged oval vortices, which are very difficult to interpret in terms of chemotaxis at each point, if viscous interaction is not assumed. In such a case, cells would have to interpret locally several scalar fields of morphogenes with antagonist actions (attractant or repellant), in order to reconstruct dipolar and quadrupolar flows, which seems contrived.

Experimentally, the core of the vortex along the "flank" of the bar has a lenticular shape with a central linear singularity (where the physical discontinuity line appears progressively), which is qualitatively the shape taken progressively by the entire limb territory. Now, the singularity in the center of the theoretical vortex takes the shape of a line following the edge of a central elongated element which behaves as a pulling area. Along this line, the speed is mathematically divergent and discontinuous : this is not observed. This is a classical problem in hydrodynamics, called Stokes-Whitehead paradox [15]. The assumption of a slow creeping flow breaks down at the point where a singular force is exerted, because of the divergence of the speed, along the discontinuity of a singular distribution of forces. In such a situation, it is known that the core of the vortex will have a speed equal to zero, and the two solutions for the flow far away from the core have to be reconnected by a smoother variation (matched expansions [15]), across a region of strong gradient; this is beyond the scope of this article. However, the classical solution for a circular vortex is the Rankine vortex core [16], for which the velocity varies linearly across the core. This is to say : the core revolves like a solid body. 
In order to resolve in the same spirit the singularity of the lenticular vortex of Equ. 3, one has to invoke a solid-body revolution in the core, which is what is observed in the chicken, as appears in Figs. 10 (data between the two stars). The experimental measurements further show that a similar simple solid-body visco-elastic revolution is the final result for the tissue behaviour in the limb plates, after the line segregating the dorsal area and the presumptive limb area has been formed, as we now show.

Indeed, as shown in Fig. 10, the presumptive area of the fold which appears along the median axis, corresponds to the area of strongest fluid shear, which corresponds to the edge of the pulling rectangle. A mathematical consequence of the physical wrinkling occuring there, is that the in-plane lateral component of the fluid is cancelled along the line (cells cannot cross). This transforms the in-plane flow away from this line into a simple drag along the line, which changes the mathematics of the problem in the following way. When integrating the dipoles along a patch of cells exerting a drag as in Equ. 3, it is assumed that all individual dipoles contribute to the flow by an individual vortex drag of the tissue. This assumes that each individual solution can propagate to the domain of integration, and all of them can be added up. However, this is no longer possible when a physical boundary, such as a wrinkle, is established. In such a case, the flow on the side located away from the line is simply dragged caudally at the edge of the wrinkle.

If we analyze finely the speed in the vortex core at the stage of Fig. 6, while it descends caudally together with the flanks, we find a remarkable situation (Figure 13(a)) : along the median axis, and up to the line of discontinuity, the flow has a parabolic profile, while in the vortex itself, the flow has a linear decay, with a sharp boundary between the two domains. This means that, once the line of discontinuity is generated along the flanks between the presumptive spine and the presumptive pelvis and limb, the presumptive spine behaves as a Poiseuille flow in a channel, and the lateral rotation is stirred like a solid-body revolution (Figure 13(b)). The parabolic profile suggests a uniform pressure drop in the median axis, oriented towards the tail, and explaining the extension of the body. One source of high pressure at one end can be ascribed to the contraction along the spine in the dorsal area, while the low pressure end is simply the free boundary in the caudal area. Another source of oriented gradient of pressure is the invaginated mesoderm which entered at the posterior pole, and, by moving anteriorily underneath, drags the ectoderm in opposite direction (i.e. posteriorily), by the principle of action and reaction. 
It is evident that the final position of the hips and of the shoulders will be defined, at least in part, by the magnitude of the winding and the position of final arrest of the "vortex" flow, as the cores drift apart.

\section{Local stress measurements.}

The viscous flow suggests that stress gradients exist in the embryo during morphogenesis, related to cell drag. Especially, if we linearize the flow around the hyperbolic point, we expect a flow map :

$$
\mathrm{v}_{\mathrm{x}}=-\mathrm{kx}, \mathrm{v}_{\mathrm{y}}=\mathrm{ky}
$$

Equ. 4

Assuming a thin film behaviour of the ectodermal sheet flowing along the mesoderm, and assuming an incompressible material, we expect the flow map of the tissue to derive from a pressure, a stress map of the form :

$$
\mathrm{P}(\mathrm{x}, \mathrm{y})=(\mathrm{k} / 2)\left(\mathrm{x}^{2}-\mathrm{y}^{2}\right) \text {, where } \mathrm{k} \text { is a dimensioning constant } \quad \text { Equ. } 5
$$

This implies that the stresses (the stress is identified with a pressure for an incompressible visco-elastic solid in thin shell with friction) should be high in the central area where the tissue is flatening, and would decrease towards the hips and shoulders and towards the tail, following the flow downstream. We have introduced recently [17] a scanning air puff tonometer (SAPT), which is able to measure such stresses in vivo. The principle of the instrument is the following: an air puff is blown on the surface and the deformation impacted on the surface is measured optically. The air puff is blown through a glass micro-pipette generated with a pipette puller. The details of the instrument can be found in Ref. 14. For measurement of the deformation, the pipette itself is used as a guide for a spot of light, shown directly inside the deformation cuvette through the pipette. By so doing, the same tip emits almost coaxially an air puff and a light spot, and the travel of the light spot during the deformation imparted by the air puff is a measurement of the total vertical displacement. We record the deflection of the spot by the NIH Image software, interfaced with the Scion frame grabber associated to the Watec 502 black and white camera. For our study, the pressure exerted at the entry of the air line were in the range 2$8 \mathrm{mbs}$, associated to a flow of the order of $0.2 \mathrm{~cm}^{3} / \mathrm{min}$, and a force of the order of $\mathrm{F} \sim 10^{-8} \mathrm{~N}$.

We analyzed by air-puff tonometry different areas of the embryos, namely: the flanks, the presumptive-hindlimb lateral plate, the presumptive-forelimb lateral plate, and the tail bud. At the stage where the lateral plate forms only a small thickening along the body (Hamilton and 
Hamburger stage 14 [18]), we found both in ovo and ex ovo that the flank area is less deformable than the lateral plates (Fig. 14(a), 14(b)), by a factor of 2.5, approximately. This discrepancy remains at later stages. When the limb bud is well formed, it is more difficult to assess the deformability in the tissue, however, by rocking the embryo up to the point that the area under study finds itself horizontal, we were able to assess the deformability by SAPT, and found again that the presumptive limb bud area is softer than the flanks in the navel area, and than the dorsal area, along the limb plate area (Fig. 14(b)).

We ascribe the deformability as related to stress, since a more stressed medium exhibits a higher resistance to surface deformations. The data show that limb outgrowth is concomitant with a localized area of smaller stress, and this stress minimum is found geometrically "above" the navel in the anterior direction, and "below the navel" in the posterior direction, in the presumptive limbs area, at a moment when they are just simple uplifts of the tissue, identified in developmental biology as the "limb plates". The stress was also found to decrease towards the apex of the tail bud (data not shown), which confirms that the caudal growth from the navel is also oriented towards decreasing stress, as expected from the Poiseuille-like profile of the speeds there.

\section{Discussion and conclusion}

This work shows that the formation of the body parts cannot be understood without adressing the viscous flow in the embryo, the associated shear and the pattern of vortices. Important flow recirculations occur by simple conservation of mass, which need not be by chemotactic navigation of cells in complex concentration gradients; a localized cell traction along the median axis suffices to oppose the viscous drag. Indeed, it is assumed in biology that cells follow chemotactic cues. However, our results show that the tissue behaves as a continuous material, which propagates at long range mechanical interactions. In this situation, the information for tissue movement need not be local : a force exerted far away, will induce complex movements, such as vortex flows, by conservation laws. Especially, simple dipolar disribution of forces will induce antagonists speeds in regions spatially close to each other, which is difficult to explain from a chemotactic point of view.

Also, this shows that the important events which set the global pattern of the animal are very rapid and counter intuitive (hyperbolic flow); the pattern of flow is then up-scaled by the dynamics of the flow itself. Therefore, the staging of embryos in classical embryology [18] is much too coarse in time, and even conceptually misleading because a continuous flow is not a 
matter of discrete stages, and the pattern is not established point-by-point. At the "stage" when limbs grow out, actually most of the patterning has already occurred earlier on by a singular vector field. This work shows in particular that the generally accepted view that embryo development is a sequence of steps organized in an "antero-posterior" order, i.e. from head to tail, is just wrong. The mechanism of tetrapod embryo formation is summarized in the Fig. 15.

Also, this work shows that the out-of-plane deformations are triggered progressively, likely as a consequence of in-plane stresses, and that a folding occurs first along the median axis, right in front of the apex of the primitive streak, induced by the highest values of the shear, which segregates the lateral plates and the median axis. The observed flow suggests a threshold shear rate for the viscous behaviour of the order of $0.2 / \mathrm{min}$. This dynamic flow is driven by internal stress gradients, generated by cellular pulls oriented one way on top, and the other way underneath.

This work, of course, is not contradictory with genetic and biomolecular analyses of the problem. Especially, it is well known that one can induce extra limbs by planting artefacts along the flanks. Generally such an artefact is a bead of plastic soaked in some chemical [19]. This may induce the erroneous idea that limbs might be grown anywhere naturally. However, if one may induce a limb anywhere by art, that does not mean that nature would be able to induce a limb anywhere by its own means. These animal assays of limb development lack a clear explanation of what is "the bead" in the normal instance. The work presented here suggests that the natural "bead" is the core of a hydrodynamic vortex. Other gross anatomical patterns might be explained by the magnitude of the vortex flow. In this view, the positioning of the limbs is a dynamical phenomenon, which, if arrested or continued, drags simply the points of outgrowth closer or farther away from the presumptive navel, following streamlines of a generic flow, at first order hyperbolic. Actually, the movies clearly show that the physical flow of tissue is, from the start, much more organized than what is usually described, and that there exists a structure for the pelvis, the shoulder girdle and limb buds at quite early stages. Also, these findings correlate with the biological observation that modifying artificially the genetics of limb formation often leads to simple anterior or posterior shifts of the point of limb outgrowth along the Anterio-Posterior axis, but not, for example, a jump of the limb on the back or on the gut [20].

It has not escaped to the authors that bipedalism might be induced by a stronger or weaker vorticity of the pelvic area which winds the lateral tissue, and positions the femur more 
or less caudally. It is tempting to suggest that genetic control of cellular tractions at the molecular level, is upscaled by the flow pattern into a pelvic rotation which has a long ranged organization. This may explain why the change from simian-like pelvis to human pelvis may appear as a simple dynamic change over a generic movement, often called "heterochrony" in paleontology [21]. More specifically, when one compares the simian pelvis and the human pelvis, it is obvious that the iliac bones are more rotated, with an engulfment oriented caudally along the sacral bone (Figure 16). A very simple explanation is that the caudal extension of the sacral vertebrae have pulled upon the lateral wings of the iliac bones. By so doing, the simian pelvis, which is quite elongated, might have become more warped (Figure 16). Of course, such a rotation needs to provide an evolutionary advantage (increased fitness), to be selected for good.

\section{APPENDIX}

\section{Materials and methods}

Chicken embryos were incubated in a standard Minitüb incubator, maintained at $38{ }^{\circ} \mathrm{C}$. The embryos were filmed through a glass window with a B\&W Watek 512 analog camera interfaced with the Scion Image software adapted from Wayne Rasband's NIH Image. In order to get better images, the embryos were entirely removed from the shell, and the yolk was carefully rinsed away. The embryos were then placed in a Petri dish in PBS buffer with additional fetal-calf serum. Embryos could be cultured this way for up to 3 to 8 hours prior to showing signs of starvation. The Movie 1 spans $5 \mathrm{hrs}$ of development filmed with a time resolution of 30secs. The stage corresponds to the end of the primitive streak formation (still involuting along the mid-line), and the very beginning of the recession of the apex. Movie 2 spans 3 hours of development it corresponds to the recession of the apex and the formation of "Hensen's node. The formation of the chord is also visible. The Movie 3 spans 4hrs and 20 minutes, filmed with a time resolution of 1 minute, it shows the winding of the neural folds, and the formation of the caudal half. The narrowing in the central part of the embryo corresponding to the presumptive navel is quite visible. The Movie 4 spans $3 \mathrm{hrs}$ and 10min. filmed with a time resolution of 1 minutes, it shows the first recognizable body at the 5 somites stage. The head with the brain folds is visible in the upper half, the central hyperbolic point is visible, the hindlimb plate revolution is very conspicuous. The Movie 5 spans 2 hours filmed with a time resolution of 5 minutes, it shows a 7 somites stage embryo; the hindlimb plate 
revolution is again very conspicuous; the closure of the median folds is very visible, the formation of the segregation between the median axis and the lateral plates is very visible. The Movie 6 spans $1 \mathrm{hr}$ filmed with a time resolution of 2 minutes, it shows a later stage of hindlimb plate revolution still evidencing the rotatory behaviour of the vector field on the lateral plates. The tissue velocity was assessed by PIV (PIV tracker module courtesy of B. Abou and O. Cardoso, available upon request; custom plugins were written for the $\operatorname{div}(\mathrm{V})$ map, and for the plotting of tangent vectors).

[1] V. Fleury, Organogenesis, 2, 1, 6, (2005).

[2] V. Fleury, Revue des Questions Scientifiques, 177, 3-4, 235, (2006).

[3] V. Fleury, A. Al-Kilani, M. Unbekandt and T.-H. Nguyen, Organogenesis, 3, 1, 49, (2007).

[4] V. Fleury, Euro. Phys. Journal, Appl. Phys. 45, 30101, (2009).

[5] R. Wetzel, Vehr. physik.-med. Ges. Würzburg 40, H.5, (1924).

[6] C. Cui, X. Yang, M. Chuai, J. A. Glazier, C. J. Weijer, Dev. Biol. 284 (1) 37-47, (2005).

[7] E. A. Zamir, B. J. Rongish, and C. D. Little, PLoS Biol. 6, e247 (2008),

[8] M. Chuai and C. Weijer, HFSP, J 3 (2), 71, (2009).

[9] H. M. Phillips, Amer. Zool. 18 (1), 81, (1978).

[10] R.A. Foty, G. Forgacs, C. M. Pfleger, and M. Steinberg, Phys. Rev. Lett. 72 (14) 1994.

[11] The movies are accessible at [to be inserted] ${ }^{1}$.

[12] L. Solnica-Krezel, Curr. Biol. 13(1):R7-9, (2003).

[13] J. Wyman, Proc. Boston Soc. Nat. Hist. Vol. 1866-1868, p. 243 (1868).

[15] W P. Graebel, Advanced Fluid Mechanics (Academic Press, London, 2007).

\footnotetext{
${ }^{1}$ If the paper is accepted, a special page dedicated to the movies will be created on the server of the Laboratoire MSC.
} 
[16] D. B. Giaiotti and F. Stel, The Rankine vortex model, PhD course in Environmental Fluid Dynamics, Physics of the atmosphere, (University of Trieste, International Center for Theoretical Physics, 2006).

[17] V. Fleury, A. Al-Kilani, O. P. Boryskina, A. J. M. Cornelissen, T.-H. Nguyen, M.

Unbekandt, L. Leroy, G. Baffet, F. le Noble, O. Sire, E. Lahaye, and V. Burgaud, Phys. Rev. E 81, 021920, (2010).

[18] V. Hamburger and H. L. J. Hamilton, Morphol. 88 49-92, (1951), See also online data base http://geisha.arizona.edu/geisha/.

[19] M. J. Cohn, J. C. Izpisúa-Belmonte, H. Abud, J. K. Heath, and C. Tickle, Fibroblast growth factor induce additional limb development from the flank of chicken embryos, Cell, 80, $5,739,(1995)$.

[20] R. C. Rallis, J. D. Buono and M. P. O. Logan, Development 132, 1961, (2005).

[21] C. Berge, Heterochronic Processes in Human Evolution: An Ontogenetic Analysis of the Hominid Pelvis, American Journal of Physical Anthropology 105:441-459 (1998). 


\section{FIGURES and LEGENDS}

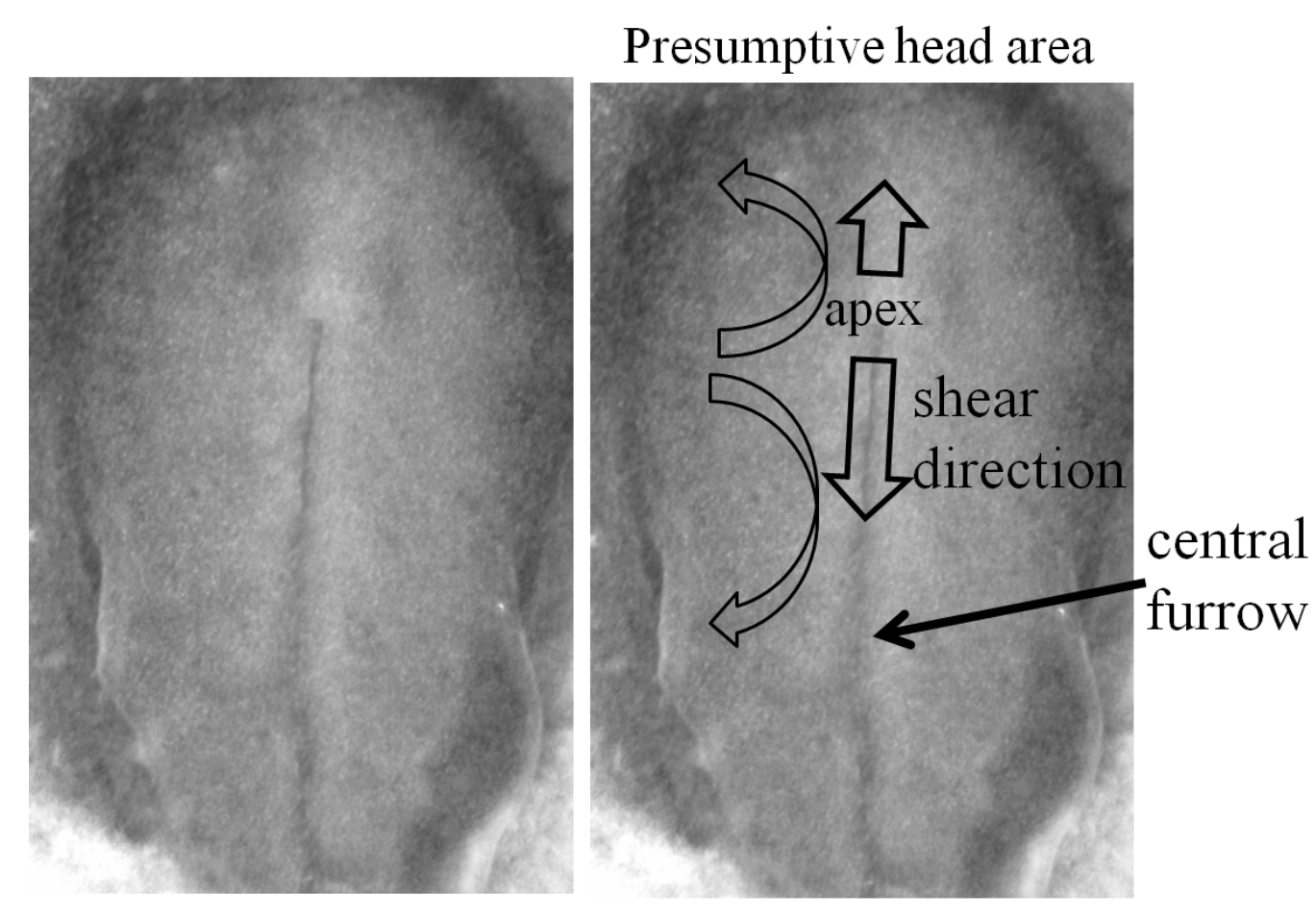

Figure 1. (Mag. 4X, full width 3mm). A chicken embryo just after the blastula stage. The flat surface contains a furrow oriented along the Antero-Posterior axis, on the midline. The apex of the furrow is called Hensen's node. It is observed in embryology that this apex moves down in the caudal direction (here to the bottom, pointed by the larger arrow), as it "heals" the furrow. As it moves, the furrow shears the lateral sides of the furrow, these are stirred and circulate with streamlines in the form of vortices. But this shear occurs on an embryo which was extending along the midline. Therefore the combination of the extension and of the downwards shear results in a hyperbolic flow. 

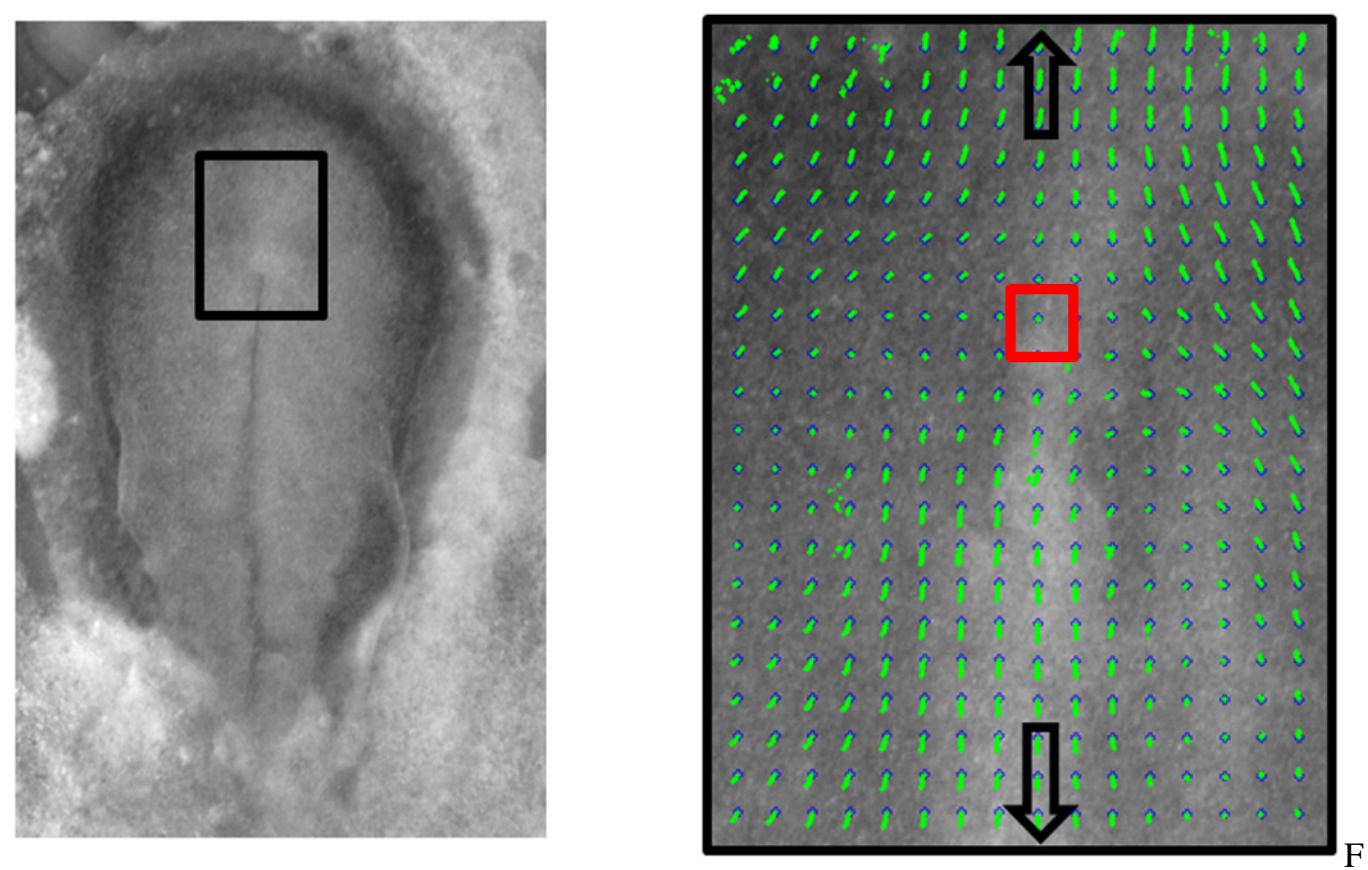

Figure 2 (Color online). 50 min. of tracking of tissue flow on the chicken embryo at an early developmental stage (1.5 days of development, PIV tracking with "tracker module" in ImageJ, same stage as Fig. 1, Movie 1 [9]). Figure 2, Left is at Mag. 4X. Fig. 2 Right shows a magnification of the box squared in the left image. The image shows the chicken gastrula while the central furrow is receding. The central furrow is visible in Fig.2 Left as a dark line along the median axis. The initial shear imparted by the extension of the median axis creates the initial pattern of hyperbolic lines. In Fig. 2, Right, the stagnation point is framed by a red square. At this point the speed is 0 in all directions. The embryo extends towards the head in the direction of the head ("cranially"), and the bottom part extends "caudally". As a result, the tissue recirculates in a pattern of streamlines which form clear circular loops over the presumptive hindlimb territory. In the area of the stagnation point the pattern is hyperbolic. 

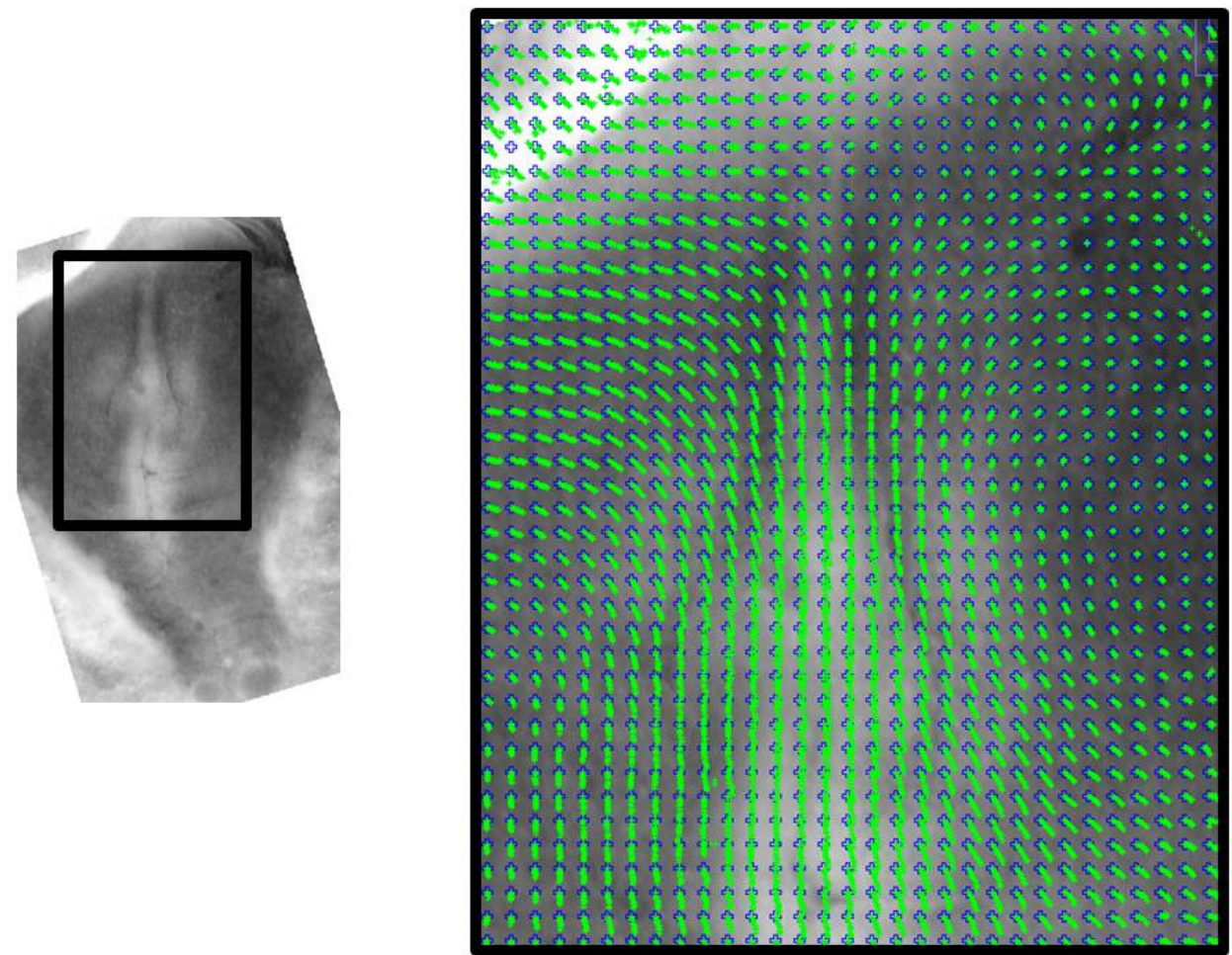

Figure 3 (Color online). (Mag. 3.8X, full height 3.9mm, Movie 2 [9]) PIV tracking of tissue flow on the embryo at a later stage (about 2 hrs after Fig. 2). At this phase of the descent of Hensen's node, the lateral tissue is obviously sheared in a pattern of vortices. There are two large vortices revolving caudally. There is a hyperbolic point separating these two large vortices from two smaller ones revolving cranially. In the caudal area one distinguishes an area which is bulging out, as it is pulled by a rectangular part located along the median axis of the embryo (the "chord"). 

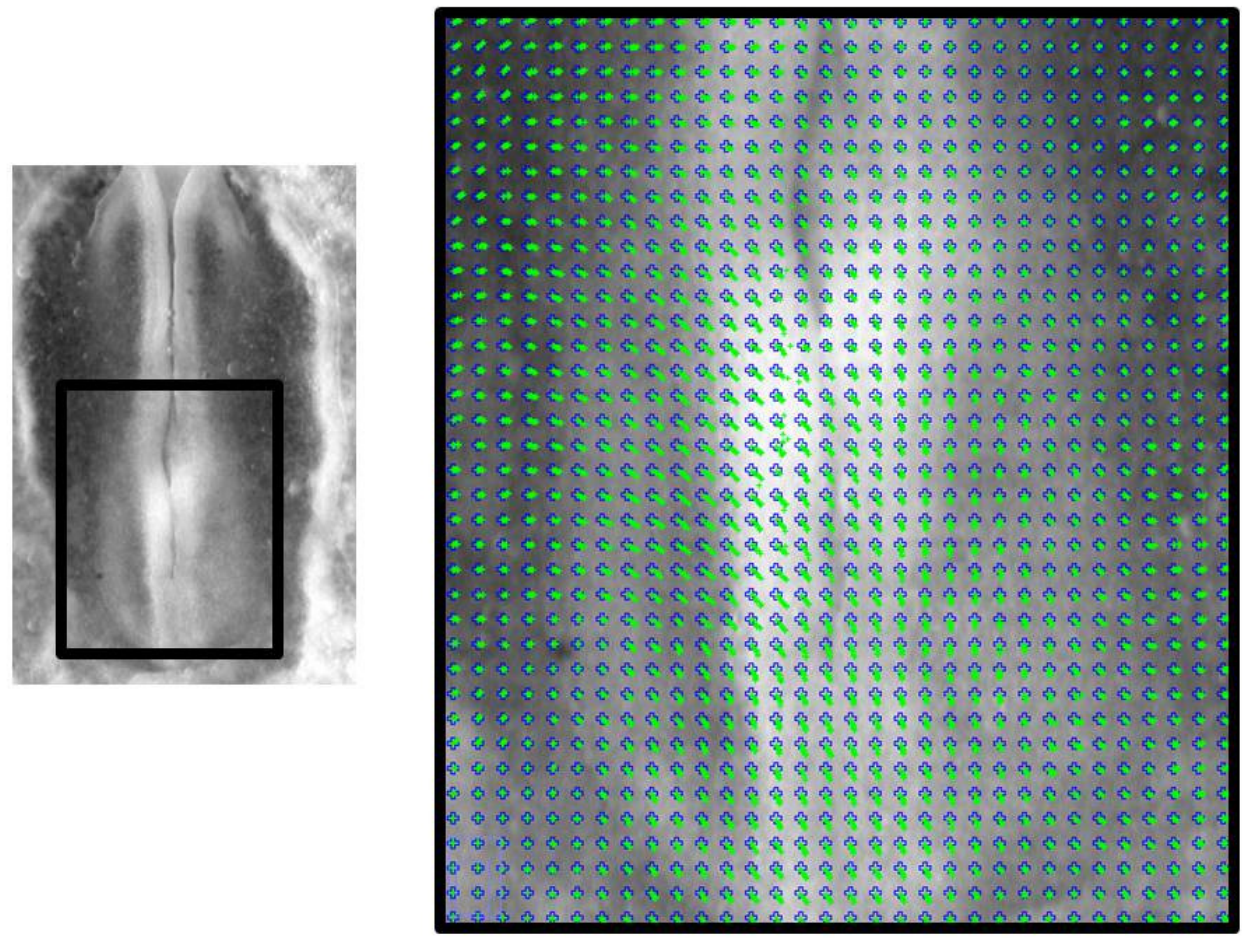

Figure 4 (Color online). (Mag. 4X, full height 2.75mm, Movie 3 [9]) As the embryo bulges more and folds, the caudal part of the embryo continues to be formed by large vortices revolving with a stagnation zone located along the sides, in the presumptive hip and limb territory. By the end of this movie, the first 3 vertebrae precursors are visible (see Movie 3, last image). 

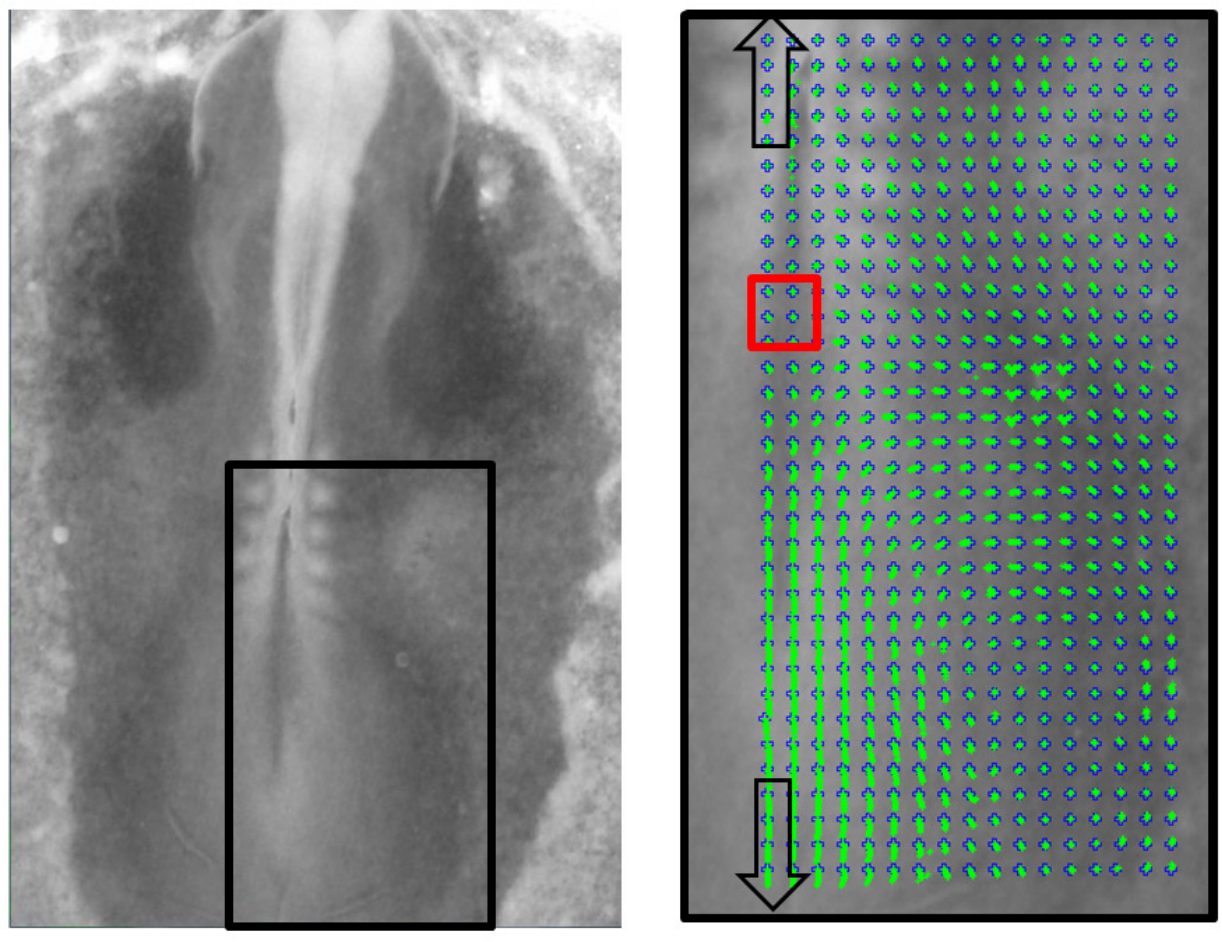

Figure 5(a) 

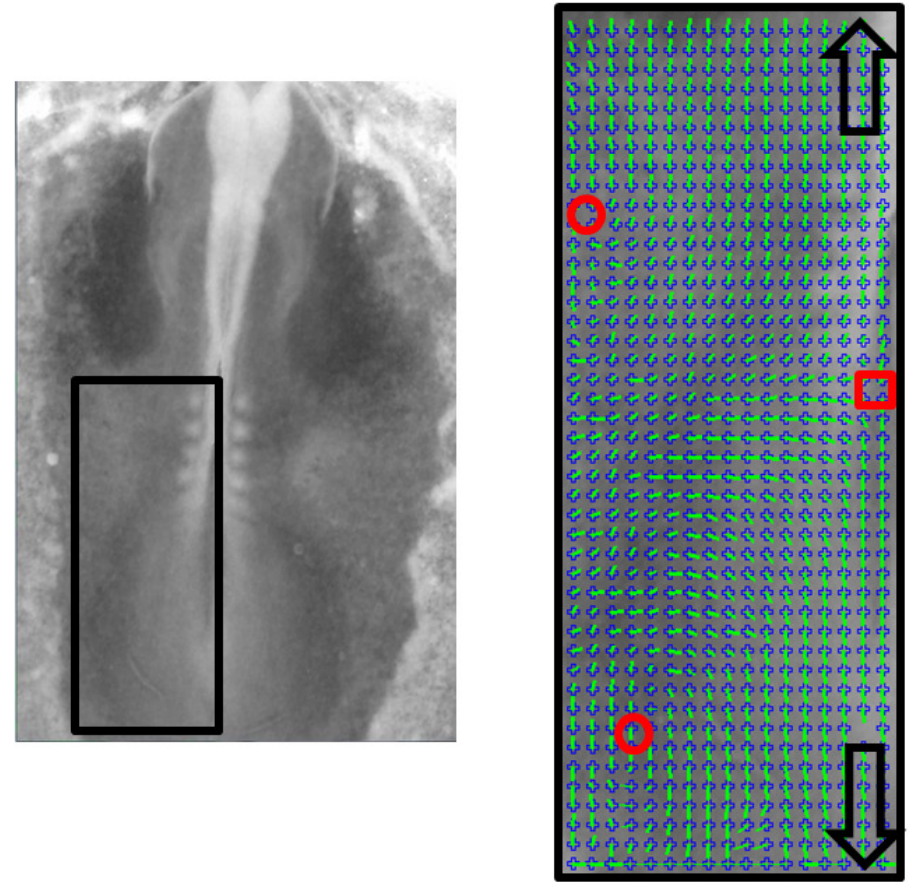

Figure 5(b).

Figure 5 (Color online). (Mag. 4X). At a somewhat later stage (approx. 4hrs after Fig. 4, Movie 4 [9]), 5 vertabrae precursors are visible (in developmental biology, the appearance of vertebrae precursors -the somites- is considered as giving the stage of development, here we see a " 5 somites stage embryo"). In the central part of the embryo, a clear stagnation point is found, and the tissue circulates in a very clear hyperbolic pattern, with a large vortex encompassing the entire hindlimb territory. Fig. 5(a) shows the vector field, with green tracks over 50 minutes of development. These tracks are extracted on the right half. Fig. 5(b) shows a similar extraction, Supprimé: in which the tangent vector to the streamline is normalized to a constant length. The red square frames the hyperbolic point, the red circles the centers of vortex-like flows. 

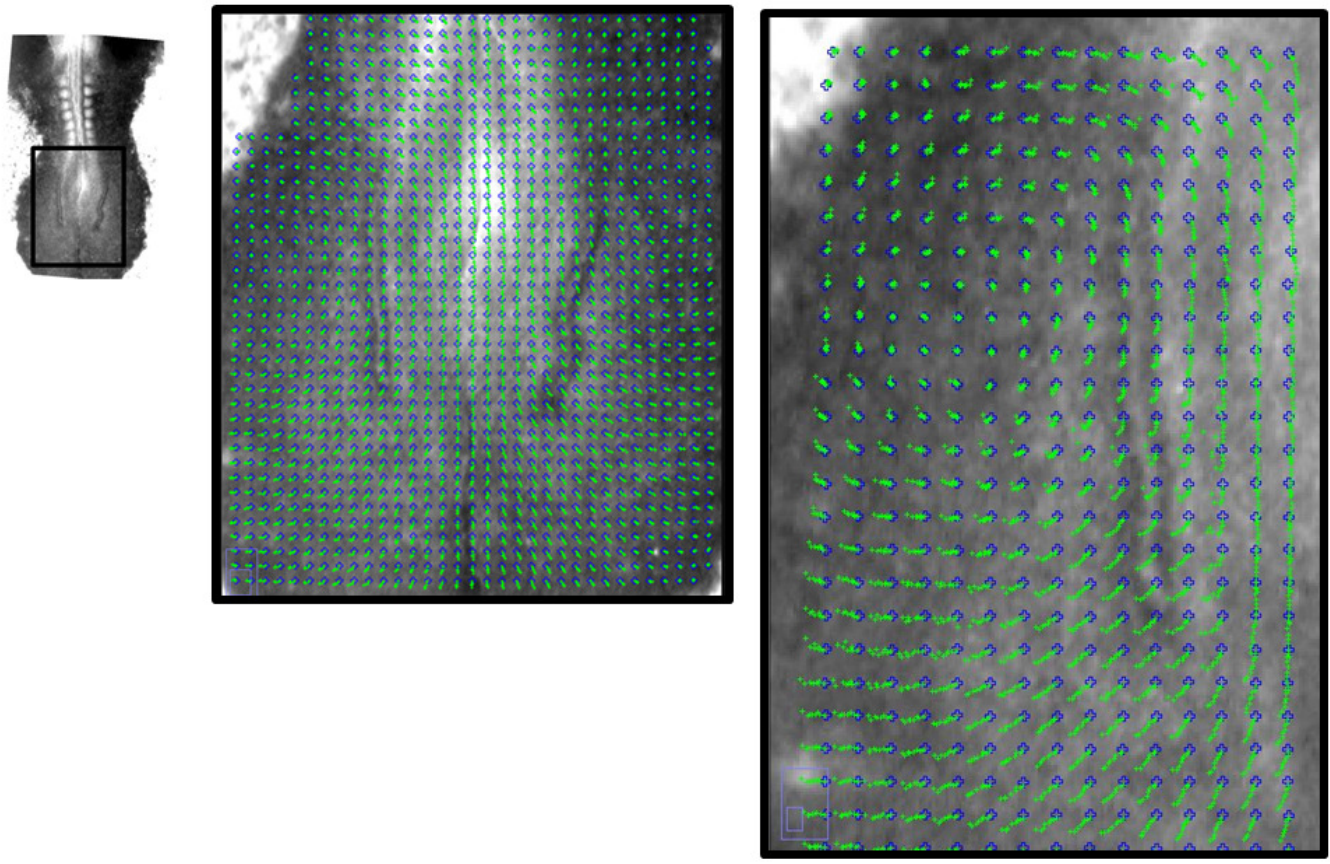

Figure 6 (a) 

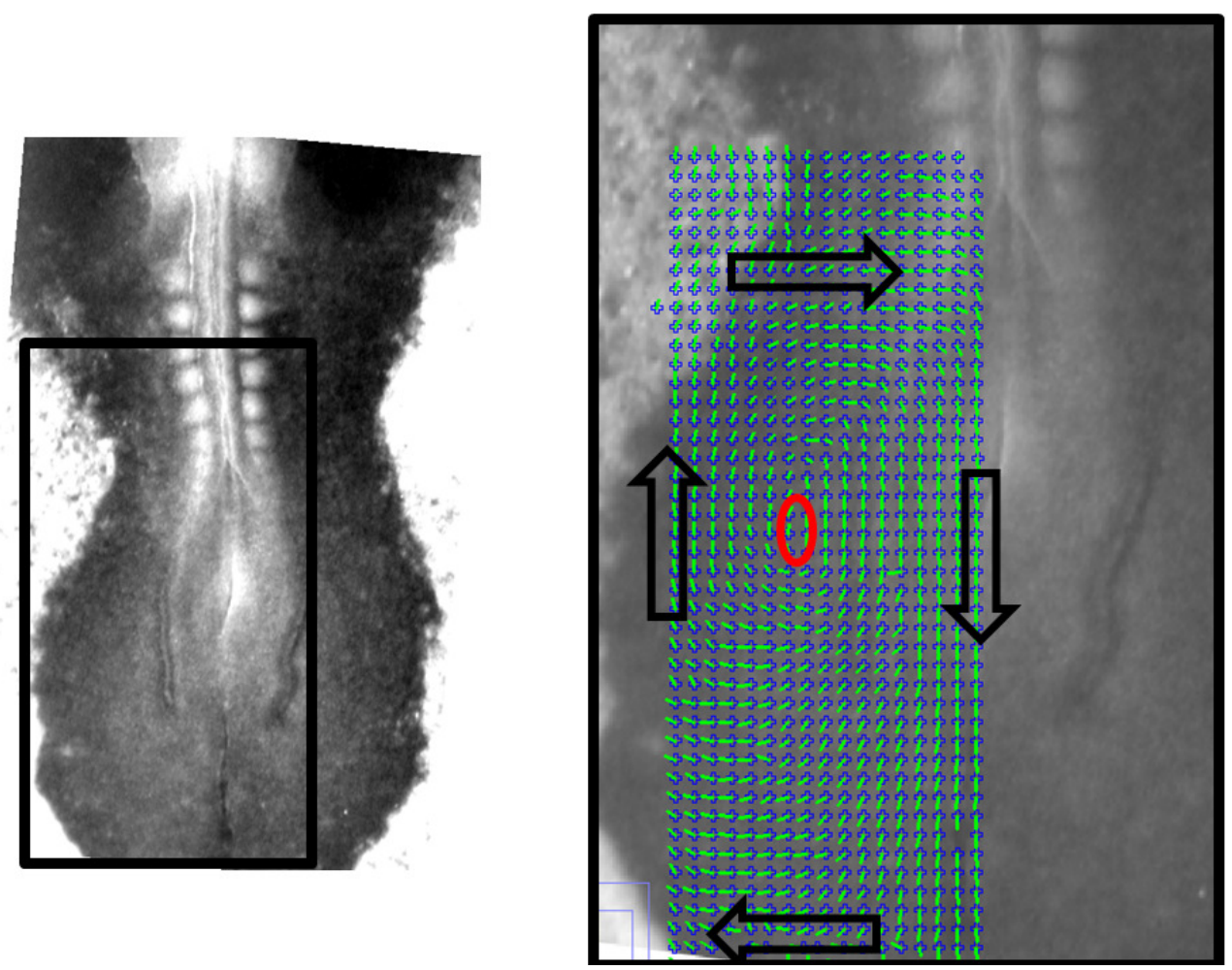

Figure 6 (Color online). (Fig. 6(a) Left: Mag. 4X, full height 2.75mm, Movie 5 [9]) 2 hours after Fig. 5, at a stage when only 7 vertebrae precursors are seen, the body keeps on extending caudally and the bulging in the hip and limb area starts to take the shape of the sacral area, with a large lenticular tissue bulging on each side of the median axis. Fig. 6(a), Middle : a magnification of the hindlimb area showing the global vortex winding. A line of discontinuity appears which separates the median area (presumptive spine and sacral area) from the lateral plate (presumptive limb and pelvis). To the right of Fig. 6(a), a magnification of the the PIV tracking shows large vortices over the hindlimb plate. Undisputable closed loops of streamlines are evidenced. Figure 6(b), the rotatory pattern is even more conspicuous on a normalized representation in which the tangent vector is represented with equal magnitude. 

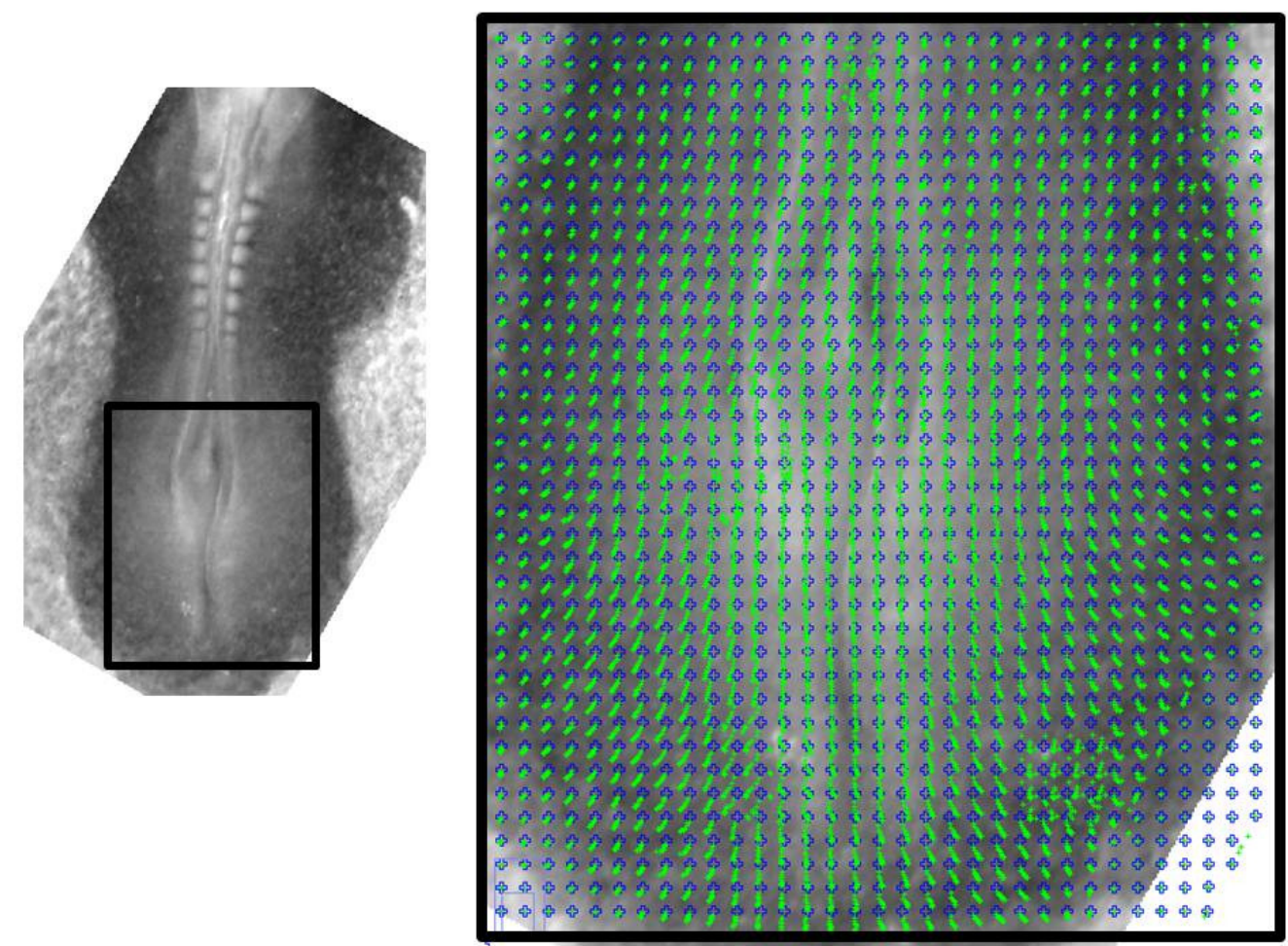

Figure 7 (Color online). (Mag. 2.8X, full height 3.9mm, Movie 6 [9]) At the stage of 8-9 vertebrae precursors, the body still extends caudally with a very large pair of vortices spanning the entire region of the hindlimbs and pelvis ( $1 \mathrm{hr}$ and 20' after Fig. 6). The caudal extension of the body is actually concomitant with the "downwards" migration of a vortex pair. 


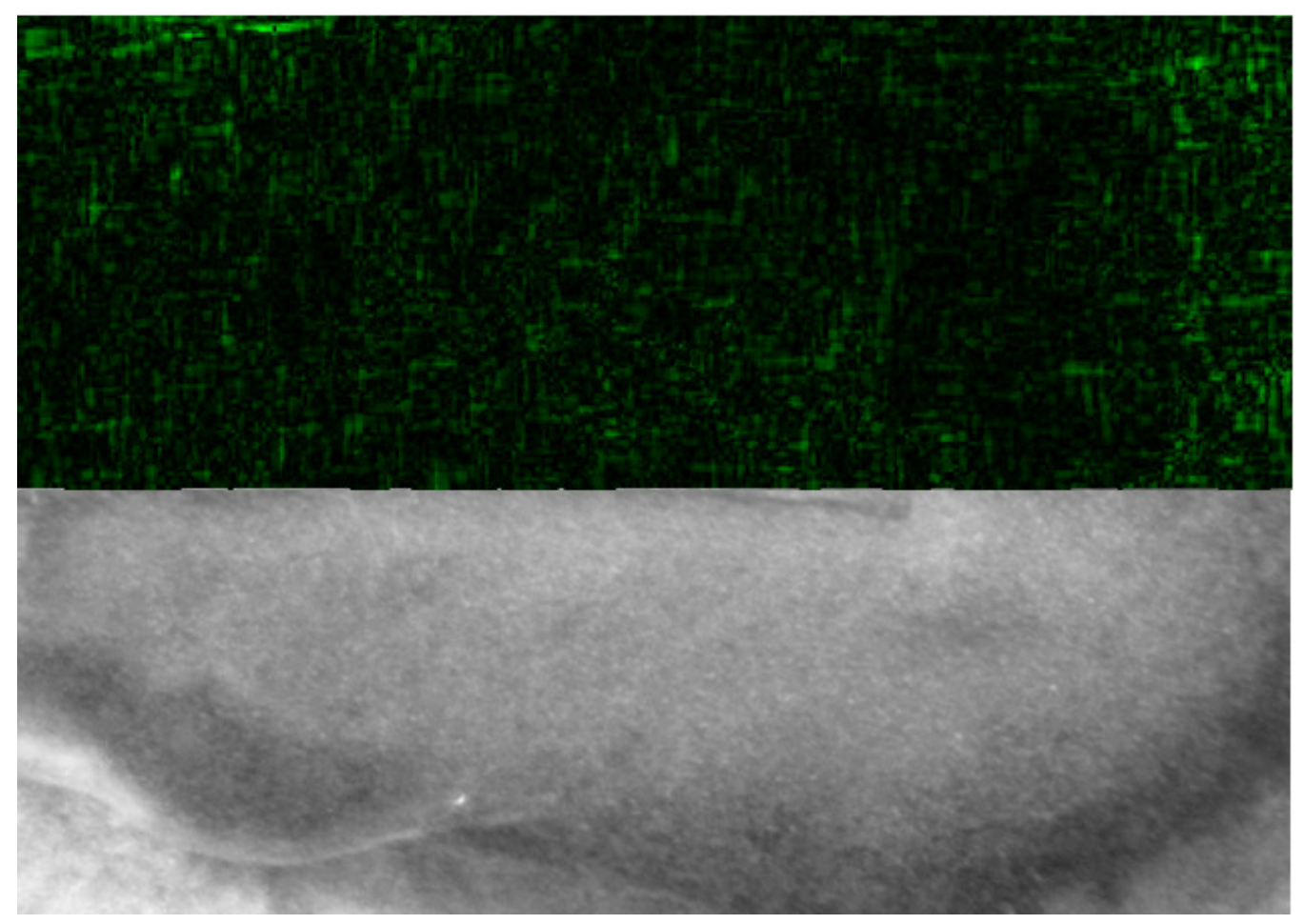

Figure 8. Extraction of the $\operatorname{div}(\mathrm{V})$ map, at the very early stage, when the embryo is almost flat (from Movie 1), shows an almost uniformly flat map of div (V). The map is shown on one half of the embryo of Fig. 2. This shows that material uptake is in a first approximation uniform. There is however a measurable departure from a uniform $\operatorname{div}(\mathrm{V})$ in the area of the apex of the furrow, likely due to the departure from perfcet flatness (this area appears darker). 


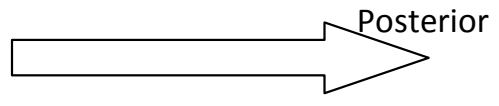

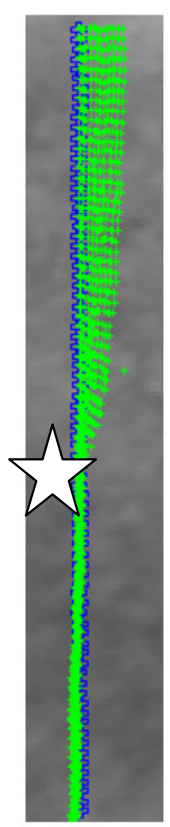

0-20

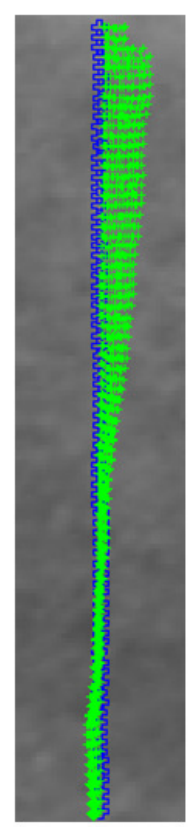

10-30

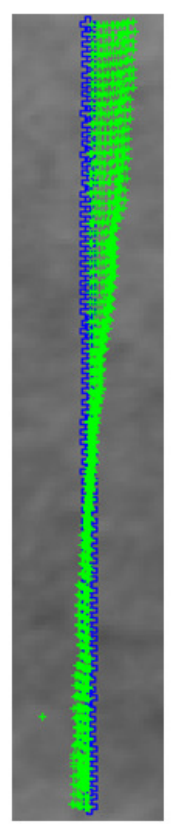

20-40

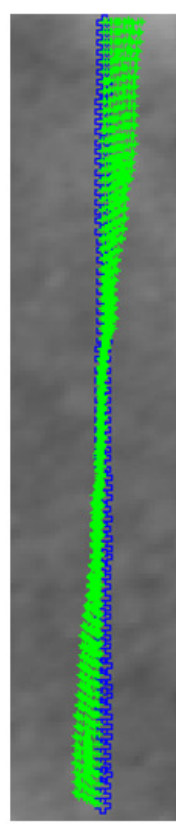

30-50
Median axis

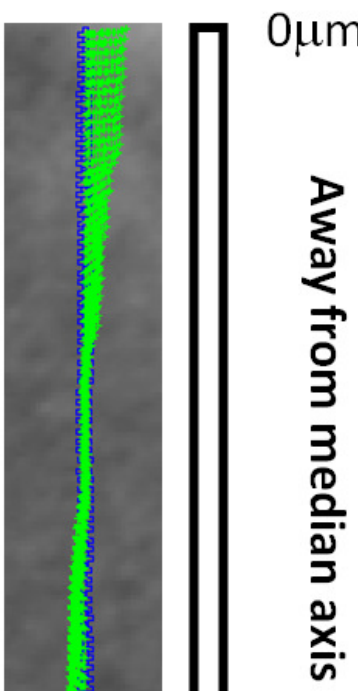

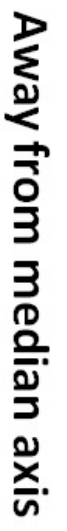

$600 \mu \mathrm{m}$

\section{Time (min.)}

Figure 9 (Color online). This plate shows one hour of analysis of velocities in Movie 2. Each box corresponds to an interval of 20 minutes. The tracks start at the blue spot and move forward along the green track. Along the median axis, the movement is always from left to right (from cranial to caudal). This finer analysis of the speeds in the area of the apex of the median folds, at start of the movement, shows that the flow is initially sheared caudally by one wide area centred along the median axis (see “0-20" minutes), but the movement has not yet propagated. At this stage, the stagnation point of the revolution is located far away from the median axis. This shear induces a rotation of the tissue, around a central core where the singularity forms. The rotation propagates in half an hour to the entire ectoderm. By one hour, the core is located much closer to the median axis, and a large vortex is formed. (These data are on the left half of the embryo, in which the singular line has not formed yet). The fact that the 
tissue rotation propagates from a state of simple shear suggests that it is not induced by chemotaxis, but by the central shear.

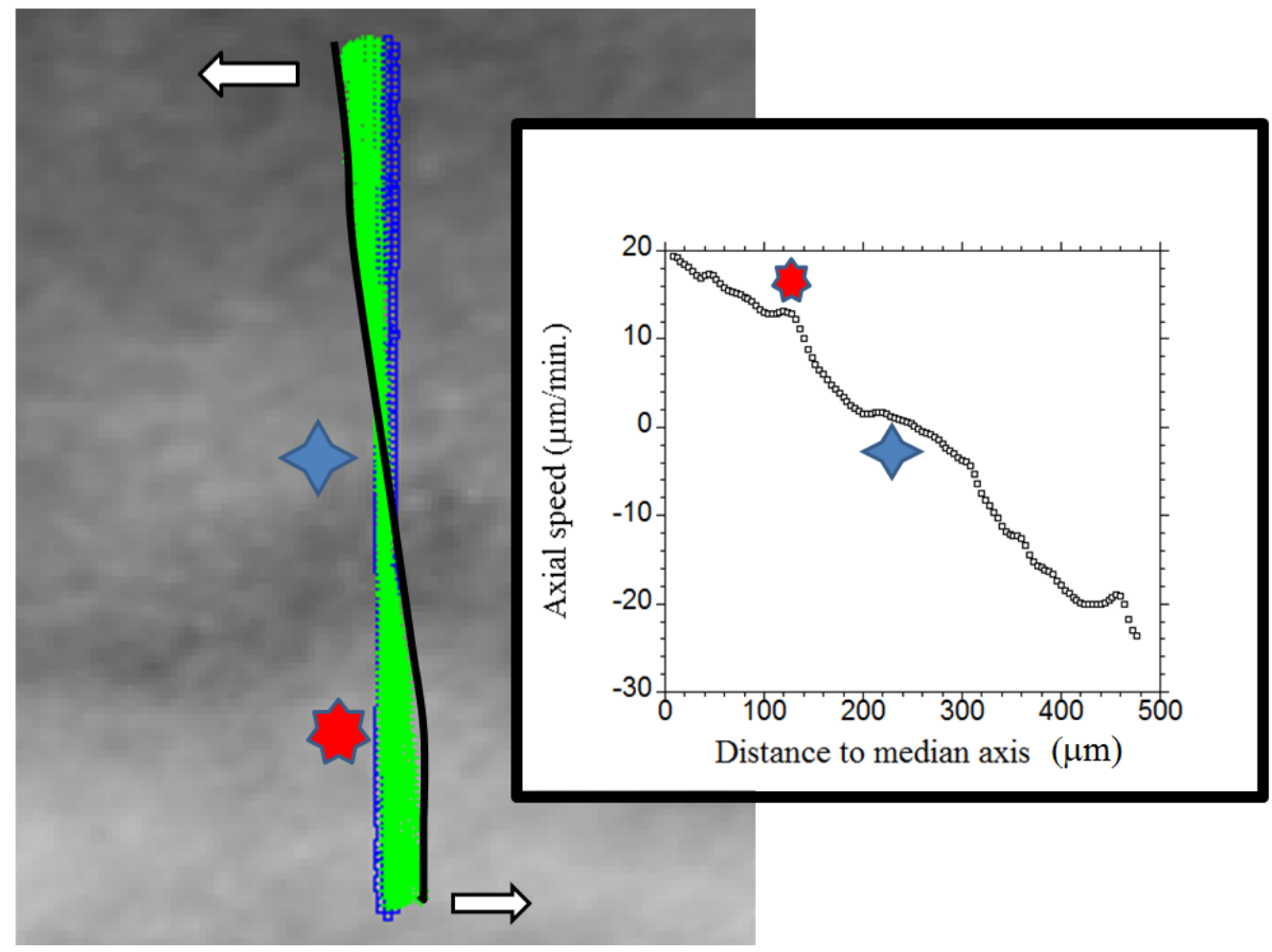

Figure 10 (Color online). This plate shows a dot by dot analysis of the speed across the lateral plate bulge, in the central part of the core of the vortex, as the singular boundary between sacral area and pelvic area is being formed (at $40 \mathrm{~min}$., with respect to Movie 2). One sees that the area comprised between the red and blue stars corresponds to the highest shear area (shear rate $0.2 /$ min.), defined as the slope $\mathrm{dV}(\mathrm{x}) / \mathrm{dy}$ of the axial speed, as a function of distance to the median axis. The area which is shearing the median axis experiences a shallow gradient (to the left of the red star in the plot), which becomes higher across the vortex (between the two stars), and reverts to smaller values away (to the right of the blue star). Far away, the larger values of the gradient are beyond this study, and may be linked to the boundary condition. As we see, the shear is indeed large around the stagnation point of the vortex (core of the vortex). 


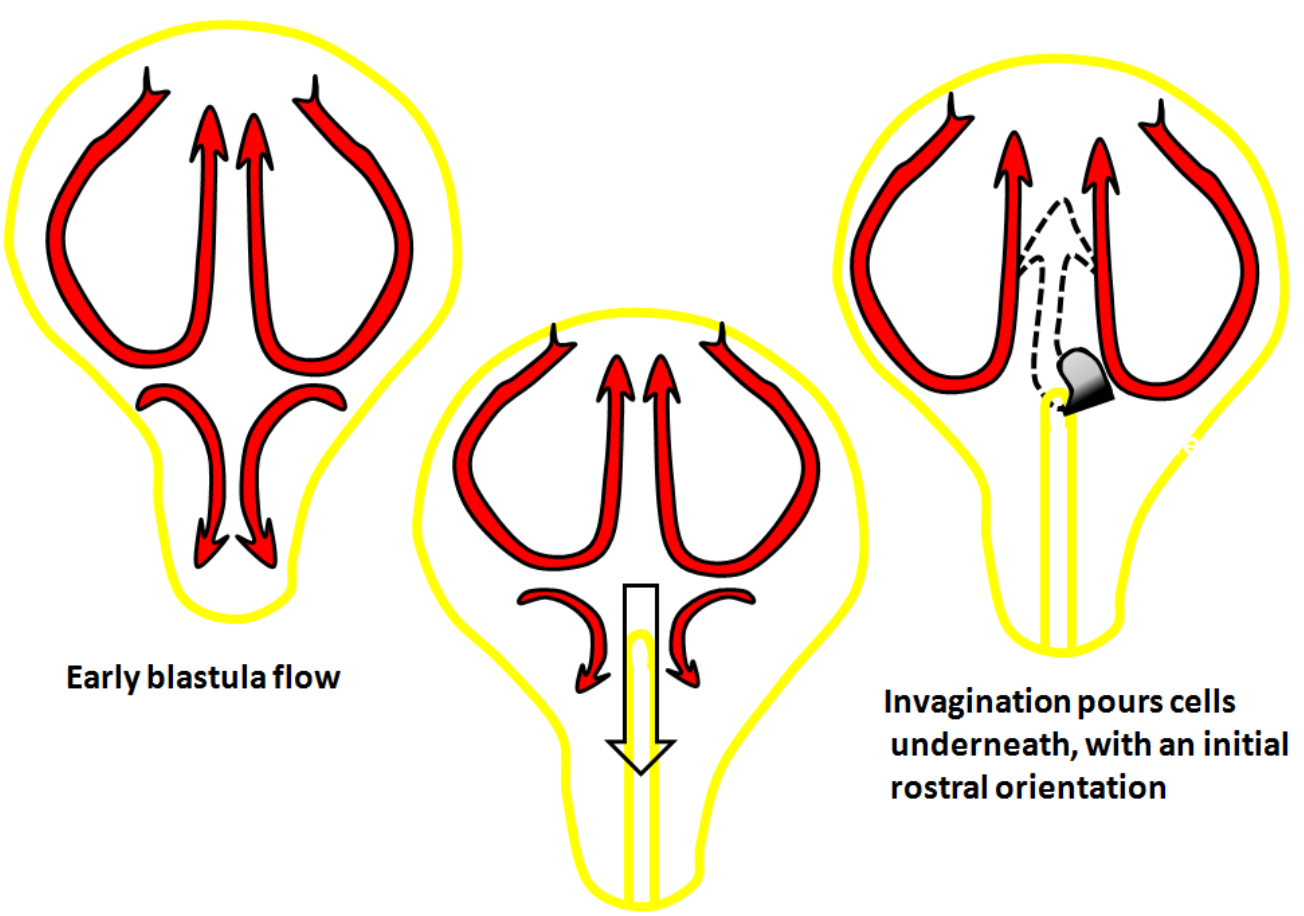

By the principle of action and reaction, the invagination adds a pull which shifts the hyperbolic point ahead of the point of invagination

Figure 11. Explanation of how the hyperbolic point appears at the apex of Hensen's node. Earlier on, the flow is hyperbolic, with a stagnation point more posterior than the furrow. The invagination of the cells (grey folded arrow in the sketch to the right) adds a strong pull at the apex of the furrow represented by the black arrow (in the sketch in the middle), which shifts the position of the hyperbolic point to a position somewhat anterior to the apex of the furrow. The origin of the initial hyperbolic flow is the so called Kohler-Rauber's Sickle [1]. There exist transient states between the different flow situations. 


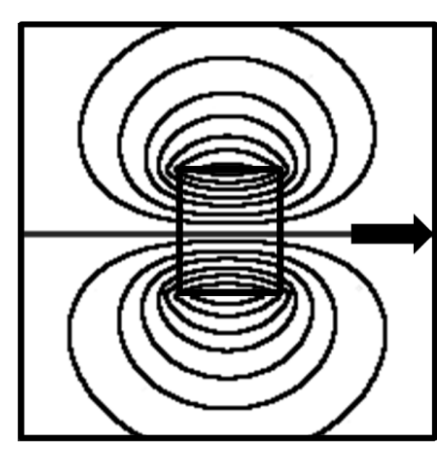

Figure 12. This figure shows the streamlines generated by a rectangle of width $2 \mathrm{~B}$, and length $2 \mathrm{~L}$, dragged across a viscous medium (the rectangle is overlaid, the forces are oriented as shown by the arrow). The result is a lenticular set of streamlines in the area of the core of the vortex, with a singular line located along the area which is pulling, ending in a discontinuous acute feature. While the stream lines have indeed an oval shape in the embryo, the velocities are not infinite, as for the theoretical vortex. It is a classical problem in linear hydrodynamics that the Stokes equation may give correct streamlines but erroneous magnitudes in the core of the vortices, because of a singular divergence in the core. 


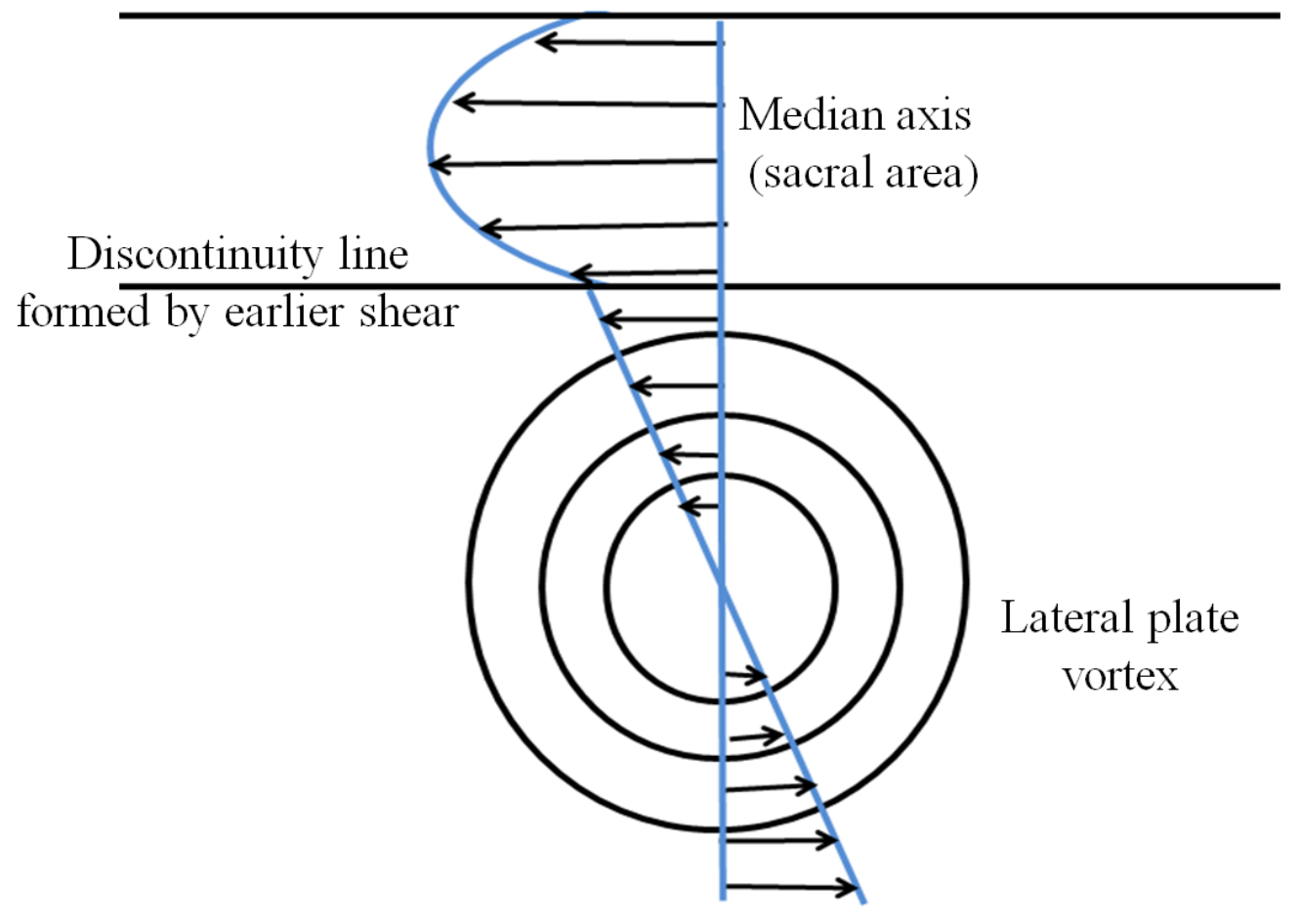

Figure 13(a) (above) 


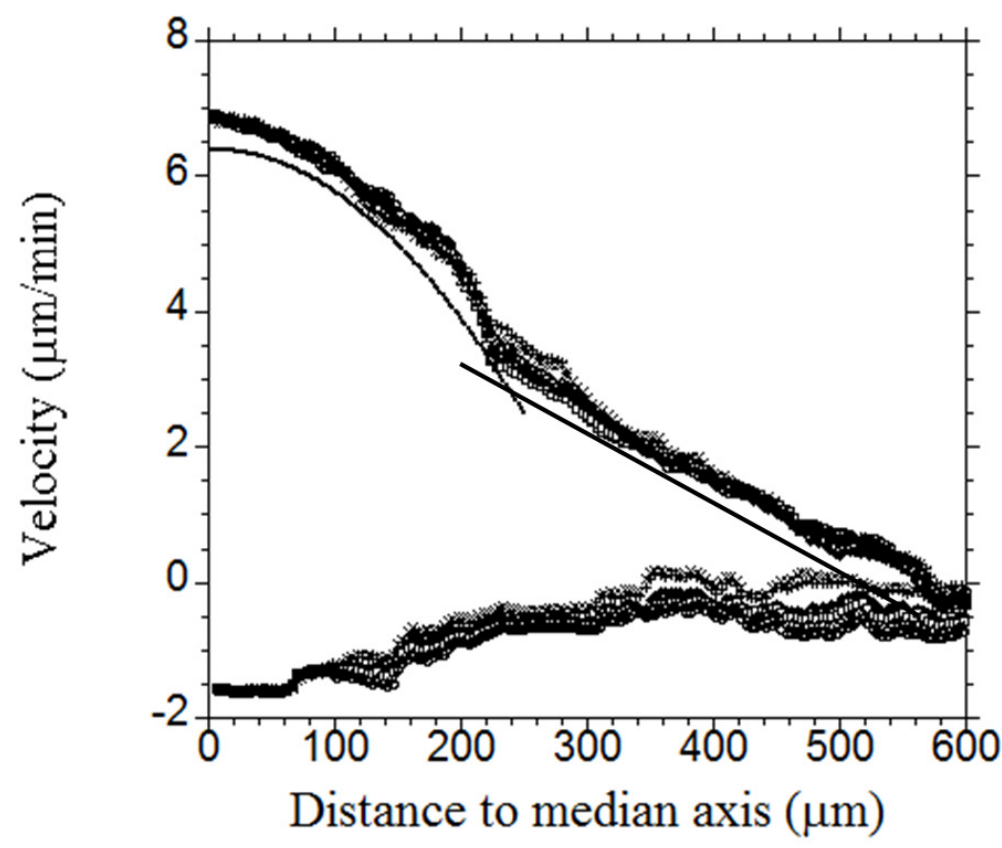

Figure 13(b) (above)

Figure 13. Figure 13(a). At later stages, schematically, the lateral plates revolves with a solid body revolution, stirred by the median axis, in which a Poiseuille like profile is observed. Figure 13(b) Shows the data collapse of the axial and orthogonal speed for 6 lines across the hindlimb area located at $\pm 8 \mu \mathrm{m}, \pm 16 \mu \mathrm{m}, \pm 32 \mu \mathrm{m}$, away from the area of the core of the vortex. The experimentally observed velocity profile can indeed be approximated by the combination of a parabolic profile in the median area between the discontinuity lines framing the axial extension, and a solid body vortex in the presumptive limb and pelvic area. The departure from a strict parabola in the median area seems to be a real fine structure, seemingly related to the fact that the central pulling area is not strictly rectangular (Hensen's node is more oval than rectangular). 


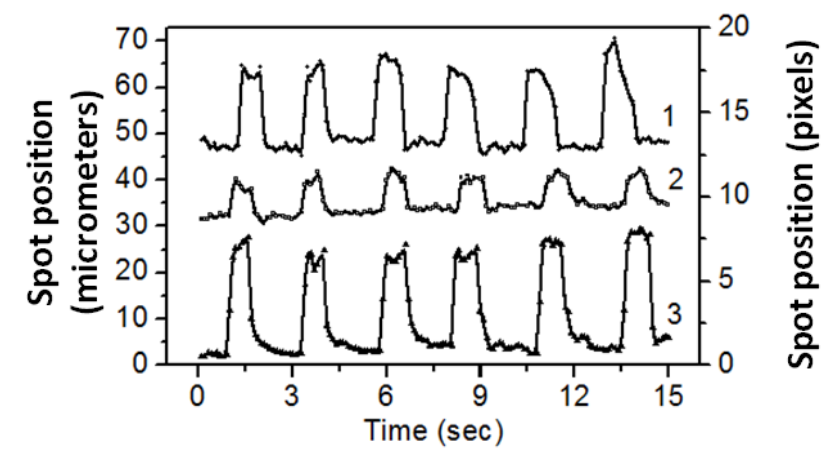

Figure 14(a) (above)

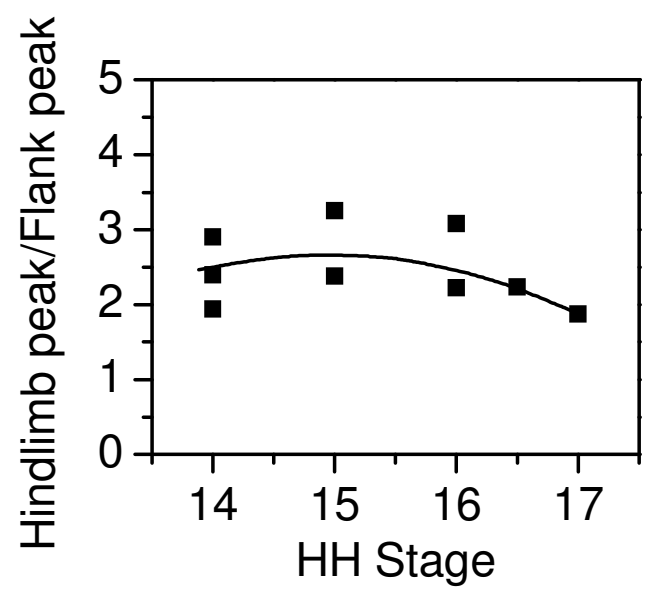

Figure 14(b) (above)

Figure 14. Figure 14(a): Position of the centre of the light spot during SAPT experiments in HH stage 14 embryo in vivo, ex ovo. The horizontal axis corresponds to time. Curve 1 stands for forelimb presumptive area, curve 2 - flank, curve 3 - hindlimb presumptive area. Data are acquired at the rate of $10 \mathrm{~Hz}$. Each air puff lasts $1 \mathrm{sec}$. During the air puff, the surface deforms in such a way that the spot moves. The deflection of the spot is recorded online. A typical measurement consists of several air puffs applied subsequently, to have a more reliable deflection signal. The data clearly show a reproducible deformation. A significant difference between flanks and presumptive limb stiffness is seen. Presumptive hindlimbs and forelimbs 
areas are, and remain, more deformable than the flanks by a factor of 2.5 , approximately during further development through stages up to 17 (Figure 14(b)).

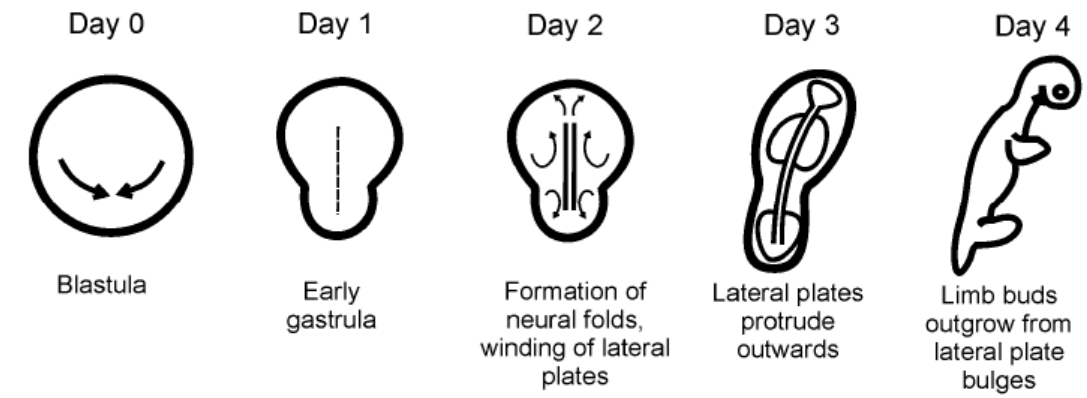

a

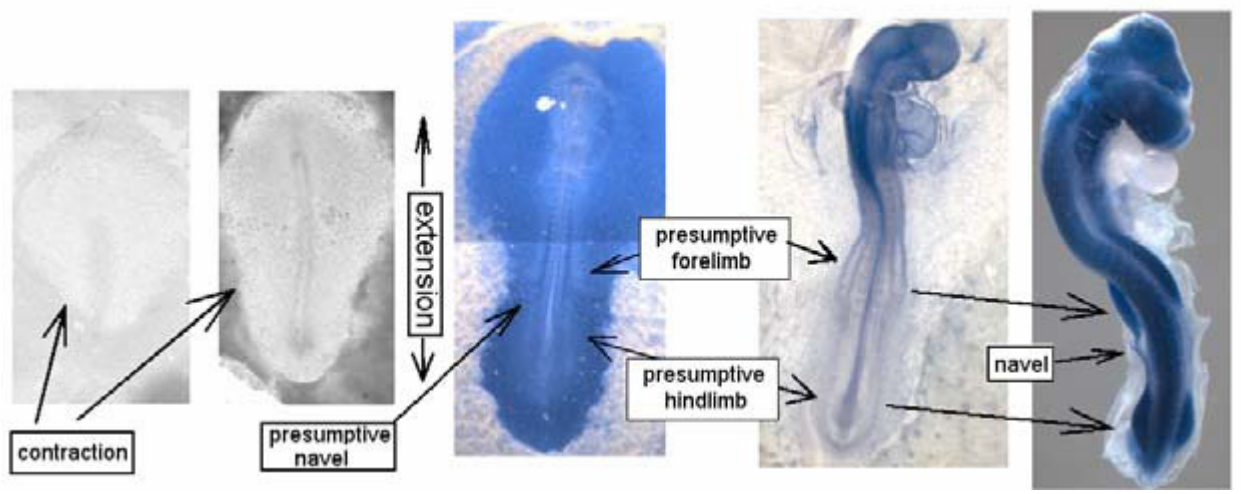

(b)

Figure 15 (Color online). Summary of the mechanism of formation of a tetrapod bauplan. Figure 15(a), scheme of the movements and the associated morphological features. Bottom Fig.15(b), typical optical snapshots of the embryos as the limb buds start to protrude (extreme right the limb buds are clearly visible, they localize exactly with the winding movements, oriented with opposite chirality with respect to the forelimbs/hindlimbs symmetry, and with respect to left/right symmetry). The stagnation point is eventually the navel. 


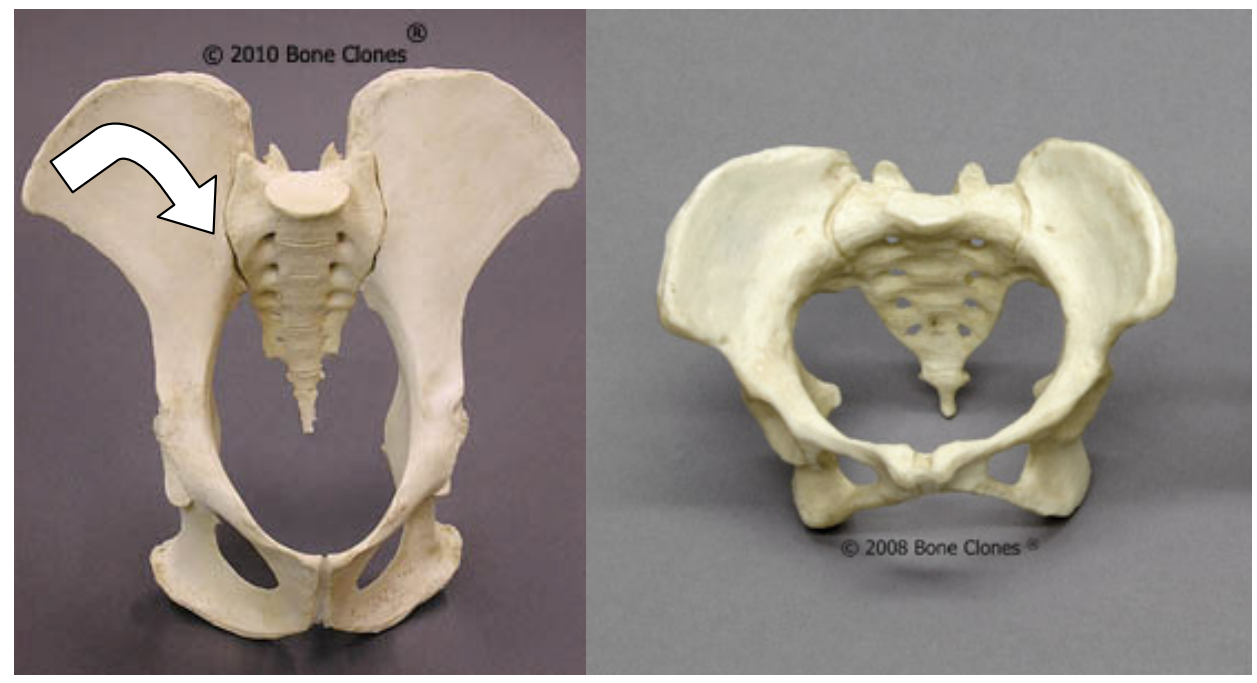

Figure 16. Left, chimpanzee pelvis. Right, human pelvis. The comparison of a chimpanzee pelvis and a human pelvis shows an apparent rotation of the illiac bone. It seems as if the sacral bone has migrated downwards in the direction of the pubic bone, dragging the lateral wings of the illiac bones in a rotatory pattern (arrow). The stronger rotation of the pelvic area ends in a more circular human pelvis, as compared to the more elongated pelvis of the chimpanzee. Of course, the chimpanzee is not the ancestor of humans, but both share a common ancestor. 
Supplementary Figure 1. 